\title{
Conferencia Mundial sobre la Educación Superior - 2009: La nueva dinámica de la educación superior y la investigación para el cambio social y el desarrollo (Sede de la UNESCO, París, 5-8 de julio de 2009)
}

\section{COMUNICADO (8 de julio de 2009)}

\section{Preámbulo}

Nosotros, los participantes en la Conferencia Mundial sobre la Educación Superior - 2009, celebrada del 5 al 8 de julio de 2009 en la Sede de la UNESCO en París, reconociendo que los resultados y la Declaración de la Conferencia Mundial sobre la Educación Superior de 1998 conservan toda su pertinencia y teniendo en cuenta las conclusiones y recomendaciones de las seis conferencias regionales (Cartagena de Indias, Macao, Dakar, Nueva Delhi, Bucarest y El Cairo), así como los debates y resultados de la presente conferencia, titulada "La nueva dinámica de la educación superior y la investigación para el cambio social y el desarrollo”, suscribimos este comunicado.

En su condición de bien público y de imperativo estratégico para todos los niveles de la enseñanza, y por ser fundamento de la investigación, la innovación y la creatividad, la educación superior debe ser responsabilidad de todos los gobiernos y recibir su apoyo económico. Como se destaca en la Declaración Universal de Derechos Humanos, “el acceso a los estudios superiores será igual para todos, en función de los méritos respectivos” (Artículo 26, párrafo 1). La recesión económica actual podría ampliar la brecha que en materia de acceso y calidad separa a países desarrollados y países en desarrollo y que se manifiesta también en el interior de los países, lo que plantearía problemas adicionales a los países donde el acceso ya está restringido.En ningún otro momento de la historia ha sido más importante que ahora la inversión en los estudios superiores, por su condición de fuerza primordial para la construcción de sociedades del conocimiento integradoras y diversas, y para fomentar la investigación, la innovación y la creatividad. La experiencia del decenio pasado demuestra que la educación superior y la investigación contribuyen a erradicar la pobreza, a fomentar el desarrollo sostenible y a adelantar en la con- 
secución de los objetivos de desarrollo acordados en el plano internacional, entre otros los Objetivos de Desarrollo del Milenio (ODM) y de la Educación para Todos (EPT). Los programas mundiales de educación deberían reflejar estas realidades.

\section{La responsabilidad social de la educación superior}

1. La educación superior, en tanto que bien público, es responsabilidad de todas las partes interesadas, en particular de los gobiernos.

2. Ante la complejidad de los desafíos mundiales, presentes y futuros, la educación superior tiene la responsabilidad social de hacer avanzar nuestra comprensión de problemas polifacéticos con dimensiones sociales, económicas, científicas y culturales, así como nuestra capacidad de hacerles frente. La educación superior debería asumir el liderazgo social en materia de creación de conocimientos de alcance mundial para abordar retos mundiales, entre los que figuran la seguridad alimentaria, el cambio climático, la gestión del agua, el diálogo intercultural, las energías renovables y la salud pública.

3. Los centros de educación superior, en el desempeño de sus funciones primordiales (investigación, enseñanza y servicio a la comunidad) en un contexto de autonomía institucional y libertad académica, deberían centrarse aún más en los aspectos interdisciplinarios y promover el pensamiento crítico y la ciudadanía activa, contribuyendo así al desarrollo sostenible, la paz y el bienestar, así como a hacer realidad los derechos humanos, entre ellos la igualdad entre los sexos.

4. La educación superior debe no sólo proporcionar competencias sólidas para el mundo de hoy y de mañana, sino contribuir además a la formación de ciudadanos dotados de principios éticos, comprometidos con la construcción de la paz, la defensa de los derechos humanos y los valores de la democracia.

5. Existe la necesidad de lograr más información, apertura y transparencia en lo tocante a las diversas misiones y actuaciones de cada establecimiento de enseñanza.

6. La autonomía es un requisito indispensable para que los establecimientos de enseñanza los puedan cumplir con su cometido gracias a la calidad, la pertinencia, la eficacia, la transparencia y la responsabilidad social.

\section{Acceso, equidad y calidad}

7. En los últimos diez años se han realizado ingentes esfuerzos a fin de mejorar el acceso y garantizar la calidad. Este empeño debe continuar. Pero el acceso, por 
si solo, no es suficiente.Será preciso hacer mucho más. Hay que llevar a cabo esfuerzos para lograr que los educandos obtengan buenos resultados.

8. La ampliación del acceso se ha convertido en una prioridad en la mayoría de los Estados Miembros y el aumento de los índices de participación en la enseñanza superior es una de las grandes tendencias mundiales. Sin embargo, aún subsisten considerables disparidades, que constituyen una importante fuente de desigualdad. Los gobiernos y las instituciones deben fomentar el acceso, la participación y el éxito de las mujeres en todos los niveles de la enseñanza.

9. Al ampliar el acceso, la educación superior debe tratar de alcanzar simultáneamente los objetivos de equidad, pertinencia y calidad. La equidad no es únicamente una cuestión de acceso - el objetivo debe ser la participación y conclusión con éxito de los estudios, al tiempo que la garantía del bienestar del alumno. Este empeño debe abarcar el adecuado apoyo económico y educativo para los estudiantes que proceden de comunidades pobres y marginadas.

10. La sociedad del conocimiento necesita diversos sistemas de educación superior, con una gama de instituciones que tengan cometidos variados y lleguen a diversos tipos de educandos. Además de los centros de enseñanza públicos, las entidades privadas de enseñanza superior con objetivos de interés público han de desempeñar una función importante.

11. Nuestra capacidad para alcanzar los objetivos de la EPT dependerá de nuestra capacidad para enfrentarnos con la escasez mundial de docentes. La educación superior debe ampliar la formación de docentes, tanto inicial como en el empleo, con planes y programas de estudios que den a los docentes la capacidad de dotar a sus alumnos de los conocimientos y las competencias que necesitan en el siglo XXI. Este objetivo exigirá nuevos enfoques, como por ejemplo el uso del aprendizaje abierto y a distancia y de las tecnologías de la información y la comunicación (TIC).

12. La formación de expertos en planificación educativa y la realización de investigaciones pedagógicas con el fin de mejorar las estrategias didácticas contribuyen también a la consecución de los objetivos de la EPT.

13. El aprendizaje abierto y a distancia y el uso de las TIC ofrecen oportunidades de ampliar el acceso a la educación de calidad, en particular cuando los recursos educativos abiertos son compartidos fácilmente entre varios países y establecimientos de enseñanza superior. 
14. La aplicación de las TIC a la enseñanza y el aprendizaje encierra un gran potencial de aumento del acceso, la calidad y los buenos resultados. Para lograr que la aplicación de las TIC aporte un valor añadido, los establecimientos y los gobiernos deberían colaborar a fin de combinar sus experiencias, elaborar políticas y fortalecer infraestructuras, en particular en materia de ancho de banda.

15. Los establecimientos de educación superior deben invertir en la capacitación del personal docente y administrativo para desempeñar nuevas funciones en sistemas de enseñanza y aprendizaje que se transforman.

16. Es esencial para todas las sociedades que se haga más hincapié en los ámbitos de las ciencias, la tecnología, la ingeniería y las matemáticas, así como en las ciencias sociales y humanas.

17. Los resultados de la investigación científica deberían difundirse más ampliamente mediante las TIC y el acceso gratuito a la documentación científica.

18. La formación que ofrecen los establecimientos de enseñanza superior debería atender las necesidades sociales y anticiparse al mismo tiempo a ellas. Esto comprende la promoción de la investigación con miras a elaborar y aplicar nuevas tecnologías y a garantizar la prestación de capacitación técnica y profesional, la educación empresarial y los programas de aprendizaje a lo largo de toda la vida.

19. La ampliación del acceso plantea un desafío a la calidad de la educación superior.

La garantía de calidad es una función esencial en la enseñanza superior contemporánea y debe contar con la participación de todos los interesados. Es una tarea que exige tanto la implantación de sistemas para garantizar la calidad como de pautas de evaluación, así como el fomento de una cultura de la calidad en los establecimientos.

20. Deberían ponerse en práctica, en todo el sector de la educación superior, mecanismos de regulación y garantía de la calidad que promuevan el acceso y creen condiciones para que los alumnos concluyan los estudios.

21. Los criterios de calidad deben reflejar los objetivos globales de la educación superior, en particular la meta de cultivar en los alumnos el pensamiento crítico e independiente y la capacidad de aprender a lo largo de toda la vida. Dichos criterios deberían estimular la innovación y la diversidad. Para garantizar la calidad en la enseñanza superior es preciso reconocer la importancia de atraer y retener a un personal docente y de investigación calificado, talentoso y comprometido con su labor. 
22. Las políticas y las inversiones deben prestar apoyo a una amplia gama de actividades de educación e investigación de tercer ciclo o postsecundarias -comprendidas las de las universidades, aunque no limitadas a ellas- $\mathrm{y}$ deben atender las necesidades que están en rápida mutación, de nuevos y diversos educandos.

23. La sociedad del conocimiento exige una diferenciación cada vez mayor de funciones dentro de los sistemas y establecimientos de educación superior, con polos y redes excelencia investigadora, innovaciones en materia de enseñanza y aprendizaje, y nuevas estrategias al servicio de la comunidad.

Internacionalización, regionalización y mundialización

24. La cooperación internacional en materia de educación superior debería basarse en la solidaridad y el respeto mutuos y en la promoción de los valores del humanismo y el diálogo intercultural. Dicha cooperación debería pues fomentarse, a pesar de la recesión económica.

25. Los establecimientos de enseñanza superior del mundo entero tienen la responsabilidad social de contribuir a reducir la brecha en materia de desarrollo mediante el aumento de la transferencia de conocimientos a través de las fronteras, en particular hacia los países en desarrollo, y de tratar de encontrar soluciones comunes para fomentar la circulación de competencias y mitigar las repercusiones negativas del éxodo de competencias.

26. Las redes internacionales de universidades y las iniciativas conjuntas forman parte de esta solución y contribuyen a fortalecer la comprensión mutua y la cultura de paz.

27. Las iniciativas conjuntas de investigación y los intercambios de alumnos y personal docente promueven la cooperación internacional. Los estímulos para lograr una movilidad académica más amplia y equilibrada deberían incorporarse a los mecanismos que garantizan una auténtica colaboración multilateral y multicultural.

28. Las iniciativas conjuntas deberían contribuir a la creación de capacidades nacionales en todos los países participantes, con lo que se garantizaría la multiplicación de fuentes de investigación homólogas de alta calidad y la generación de conocimientos, a escala regional y mundial.

29. Para que la mundialización de la educación superior nos beneficie a todos, es indispensable garantizar la equidad en materia de acceso y de resultados, promover la calidad y respetar la diversidad cultural y la soberanía nacional. 
30. La mundialización ha puesto de relieve la necesidad de establecer sistemas nacionales de acreditación de estudios y de garantía de calidad, y de promover la creación de redes entre ellos.

31. La prestación transfronteriza de enseñanza superior puede representar una importante contribución a la educación superior, siempre y cuando ofrezca una enseñanza de calidad, promueva los valores académicos, mantenga su pertinencia y observe los principios básicos de l diálogo y la cooperación, el reconocimiento mutuo, el respeto de los derechos humanos, la diversidad y la soberanía nacional.

32. La educación superior transfronteriza también puede generar oportunidades para prestatarios deshonestos y de poca calidad, cuya acción debe contrarrestarse. Los proveedores espurios (“fábricas de diplomas”) constituyen un grave problema. La lucha contra esas “fábricas de diplomas” exige esfuerzos multifacéticos de ámbito nacional e internacional.

33. Las nuevas tendencias están transformando el panorama de la educación superior y la investigación. Esta dinámica exige iniciativas conjuntas y acción concertada en los planos nacional, regional e internacional con el fin de garantizar la calidad y sostenibilidad de los sistemas de educación superior en el mundo entero - especialmente en el África subsahariana, los Pequeños Estados Insulares en Desarrollo (SIDS) y otros Países Menos Adelantados (PMA). Esto debería comprender también la cooperación Sur-Sur y Norte-Sur-Sur.

34. Sería deseable que aumentase la cooperación regional en aspectos como la convalidación de estudios y diplomas, la garantía de calidad, la gobernanza, y la investigación e innovación. La educación superior debería reflejar las dimensiones internacional, regional y nacional, tanto en la enseñanza como en la investigación.

\section{El aprendizaje y la investigación e innovación}

35. Habida cuenta de la necesidad que muchos países experimentan de disponer de más financiación para la investigación y el desarrollo, los establecimientos deberían buscar nuevas formas de aumentar la investigación y la innovación mediante iniciativas conjuntas de múltiples copartícipes entre el sector público y el privado, que abarquen a las pequeñas y medianas empresas.

36. Resulta cada vez más difícil mantener un buen equilibrio entre la investigación básica y la aplicada, debido a los altos niveles de inversión que necesita 
la investigación básica y al reto que representa el vincular el conocimiento de ámbito mundial con los problemas locales. Los sistemas de investigación deberían organizarse de manera más flexible con miras a promover la ciencia y la interdisciplinariedad, al servicio de la sociedad.

37. En aras de la calidad y la integridad de la educación superior, es importante que el personal docente disponga de oportunidades para realizar investigaciones y obtener becas. La libertad de enseñanza es un valor fundamental que es preciso proteger en el contexto internacional actual de inestabilidad y evolución constante.

38. Los establecimientos de enseñanza superior deberían buscar esferas de investigación y docencia capaces de abordar los asuntos que atañen al bienestar de la población y crear bases sólidas para la ciencia y la tecnología pertinentes en el plano local.

39. Los sistemas de conocimiento indígenas pueden ampliar nuestra comprensión de los nuevos problemas; la educación superior debería establecer asociaciones de mutuo beneficio con las comunidades y las sociedades civiles, con miras a facilitar el intercambio y la transmisión de los conocimientos adecuados.

40. Ante la creciente escasez de recursos, se insta a las partes interesadas a que estudien e intensifiquen el uso de los recursos e instrumentos de las bibliotecas electrónicas, con miras a apoyar la enseñanza, el aprendizaje y la investigación.

\section{La educación superior en África}

41. La Conferencia Mundial sobre la Educación Superior - 2009 otorgó especial atención a los problemas y las oportunidades que plantea la revitalización de la enseñanza superior en África, que constituye una importante herramienta de desarrollo en ese continente. Las cuestiones que el tema suscitó se encuentran reflejadas a todo lo largo del presente Comunicado.

42. Los participantes acogieron con beneplácito las recomendaciones de la Conferencia preparatoria regional de Dakar, celebrada en noviembre de 2008, y tomaron nota de los progresos registrados desde la Conferencia Mundial sobre la Educación Superior de 1998, en particular el aumento de la matrícula en la enseñanza superior. Los participantes subrayaron la imperiosa necesidad de hacer frente a los nuevos desafíos en lo tocante a la desigualdad de raza y género, la libertad de enseñanza, el éxodo de competencias y la escasa preparación de los graduados cuando acceden al mercado laboral. También insistieron en la urgente 
necesidad de impulsar una nueva dinámica en materia de educación superior en África, que tienden a una amplia transformación con el fin de aumentar considerablemente su pertinencia y capacidad de respuesta a las realidades políticas, sociales y económicas de los países del continente. Este nuevo impulso podría reorientar los esfuerzos que se llevan a cabo para combatir el subdesarrollo y la pobreza en África. Para ello sería necesario prestar más atención a la educación superior y la investigación en el continente de la que se le ha brindado en los últimos once años. La educación superior en África debería fomentar la gobernanza basada en una clara atribución de responsabilidades y en sólidos principios financieros.

43. El ámbito africano de educación superior e investigación ganará en calidad mediante la colaboración institucional, nacional, regional e internacional. Por consiguiente, es necesario disponer de una orientación estratégica que permita la creación o el fortalecimiento de dicha colaboración. Los países africanos que cuentan con sistemas de enseñanza superior desarrollados deberían intercambiar experiencias con sus homólogos que han alcanzado un menor grado de desarrollo. Debemos comprometernos a que la educación superior en África funcione como un instrumento de integración regional.

44. La creación de un mecanismo de garantía de calidad de alcance regional tendrá también un efecto catalizador en el desarrollo de la esfera de la educación superior en África. En este sentido, exhortamos a que se aplique rápidamente la iniciativa de la Asociación de Universidades Africanas (AUA) que, con el apoyo de la UNESCO, estimulará la creación de sistemas de garantía de calidad nacionales, subregionales y regionales. Asimismo, la movilidad del alumnado y el personal docente dentro del ámbito africano de educación superior será fomentada mediante la aplicación del Convenio de Arusha sobre la convalidación de diplomas, certificados y títulos. También se debería hacer hincapié en que la labor de la Universidad Panafricana ES indispensable para fomentar la integración del continente.

45. Acceso: Con el fin de satisfacer el rápido aumento de la demanda de educación superior e investigación, África necesita urgentemente instituciones diferenciadas, en una gama que vaya de las universidades de investigación a los politécnicos e institutos de tecnología, con programas diversificados en cada tipo de establecimiento, adaptados a los distintos tipos de educandos y a las necesidades de cada país. La demanda cada vez mayor de enseñanza superior no podrá satisfacerse únicamente con las actividades tradicionales del magisterio 
presencial. Será preciso utilizar otras estrategias, como la enseñanza abierta y a distancia y el aprendizaje en línea, especialmente en esferas como la educación permanente de adultos y la formación de docentes.

46. Pertinencia de los planes y programas de estudios: Determinados ámbitos de competencia que revisten una importancia fundamental para la diversificación de las economías africanas no reciben actualmente la atención debida. Entre otros, cabe citar a la agricultura, la extracción de recursos naturales, el medio ambiente, los sistemas de conocimiento indígenas y la energía. Si la enseñanza superior prestara más atención a estas esferas, contribuiría a garantizar la competitividad de las economías del continente.

47. Financiación: La educación sigue siendo un bien público, pero debería estimularse también la financiación privada. Aunque deben agotarse todos los esfuerzos con vistas a aumentar la financiación pública de la educación superior, es preciso reconocer que los fondos públicos son limitados y tal vez no resulten suficientes para un sector que evoluciona tan rápidamente. Deberían explorarse otras fuentes de financiación, en particular las que se basan en iniciativas conjuntas de los sectores público y privado.

48. Debería tenerse en cuenta la opinión de los educandos sobre la gobernanza en todos los niveles de la educación superior.

49. Los participantes expresaron su profunda gratitud a diversos países y organizaciones por el apoyo permanente que proporcionan al desarrollo de la educación superior en África. También acogieron con beneplácito las nuevas promesas formuladas por otros asociados, en particular China, la India y la República de Corea. Asimismo aplaudieron las propuestas específicas del Banco Africano de Desarrollo, la Unión Africana, y las asociaciones de universidades -en particular la Asociación de Universidades Africanas (AUA), la Agencia Universitaria de la Francofonía (AUF) y la Asociación de Universidades del Commonwealth (ACU)- sobre los temas de la gobernanza y los modelos de enseñanza superior.

50. Los participantes apreciaron la prioridad que la UNESCO otorgó al África en esta conferencia.

\section{Llamamiento a la acción: los estados miembros}

51. Los Estados Miembros, en colaboración con todas las partes interesadas, deberían elaborar políticas y estrategias en el plano de los sistemas y de los establecimientos con el fin de: 
a) mantener y, si fuera posible, aumentar la inversión en el sector de la educación superior para apoyar en todo momento la calidad y la equidad y fomentar la diversificación, tanto en la prestación de servicios de enseñanza superior como en los medios de financiarla;

b) garantizar las inversiones adecuadas en la educación superior y la investigación, em sintonía con las necesidades y expectativas crecientes de la sociedad;

c) establecer y fortalecer, con la participación de todos los interesados, los sistemas de garantía de calidad y los marcos normativos apropiados;

d) ampliar la formación de docentes, tanto inicial como en el empleo, con programas que les capaciten para hacer de sus estudiantes ciudadanos responsables;

e) alentar el acceso, la participación y los buenos resultados académicos de las mujeres en la enseñanza superior;

f) garantizar la igualdad de acceso a los grupos insuficientemente representados, tales como los obreros, los pobres, las minorías, las personas con capacidades diferentes, los migrantes, los refugiados y otros grupos de población vulnerables;

g) crear mecanismos que permitan contrarrestar las repercusiones negativas del éxodo de competencias y, al mismo tiempo, estimulen la movilidad de los docentes, los estudiantes y el personal de educación;

h) apoyar el aumento de la cooperación regional en materia de enseñanza superior con miras a la creación y el fortalecimiento de ámbitos regionales de educación superior e investigación;

i) dotar de autonomía a los Países Menos Adelantados y a los Pequeños Estados Insulares en Desarrollo para que puedan aprovechar las oportunidades que ofrece La mundialización, y fomentar la colaboración entre ellos;

j) tratar de alcanzar los objetivos de equidad, calidad y éxito académico mediante la creación de vías de acceso más flexibles y una mejor convalidación del aprendizaje previo y la experiencia laboral;

k) aumentar el atractivo de las carreras académicas, garantizando el respeto de los derechos y las condiciones de trabajo adecuadas del personal docente, según lo estipulado en la Recomendación de 1997 relativa a la condición del personal docente de la enseñanza superior;

l) lograr la participación activa de los estudiantes en la vida académica, garantizándoles la libertad de expresión y el derecho a organizarse, y facilitándoles los servicios adecuados; 
m) luchar contra las “fábricas de diplomas” mediante iniciativas multifacéticas en la esfera nacional e internacional;

n) crear sistemas de investigación flexibles y organizados, que promuevan la excelencia científica y la interdisciplinariedad y sean útiles para la sociedad;

o) apoyar una mayor integración de las TIC y fomentar el aprendizaje abierto y a distancia, con miras a satisfacer el aumento de la demanda de educación superior.

\section{Llamamiento a la acción: la UNESCO}

52. En el contexto de los progresos importantes realizados en la consecución de la educación primaria universal, la UNESCO debería reafirmar la prioridad de la educación superior en sus programas y presupuestos futuros. Para hacer realidad esta prioridad en el marco de sus cinco funciones de laboratorio de ideas, catalizador de la cooperación internacional, organismo normativo, creador de capacidad en los Estados Miembros y centro de intercambio de información, la UNESCO debería en particular:

a) prestar asistencia en la formulación de estrategias sostenibles y a largo plazo para la educación superior y la investigación, en sintonía con los objetivos de desarrollo acordados internacionalmente y las necesidades nacionales o regionales;

b) facilitar estructuras para el diálogo y el intercambio de experiencias e informaciones sobre la educación superior y la investigación, y contribuir al aumento de capacidades para la elaboración de políticas relativas a la educación superior y la investigación;

c) ayudar a los gobiernos y las instituciones para que aborden los problemas internacionales en la esfera de la educación superior mediante:

- la aplicación permanente de sus instrumentos normativos, en particular la nueva generación de convenios regionales de convalidación de estudios, y la Recomendación de 1997 relativa a la condición del personal docente de la enseñanza superior;

- la continuidad de su labor de aumento de capacidades con miras a garantizar la calidad en materia de educación superior en los países en desarrollo;

- el fomento de la colaboración internacional en la formación de docentes en todas las regiones, particularmente en África, por conducto de la Iniciativa para la Formación de Docentes en el África Subsahariana (TTISSA); 
- el estímulo a la transferencia de conocimientos mediante las redes UNITWIN y de Cátedras UNESCO, en colaboración con otros organismos, a fin de promover el aumento de capacidades para apoyar la consecución de los objetivos internacionales de desarrollo, tales como la Educación para Todos (EPT), los Objetivos de Desarrollo del Milenio (ODM) y los de los Decenios de las Naciones Unidas;

d) estimular la movilidad y los intercambios internacionales de alumnos y personal docente, y al mismo tiempo elaborar estrategias para contrarrestar las consecuencias negativas del éxodo de competencias;

e) fortalecer la participación de los estudiantes en los foros de la UNESCO y apoyar El diálogo entre los educandos de todo el mundo;

f) velar por el seguimiento de la Conferencia Mundial sobre la Educación Superior - 2009, por medio de la definición de los temas y las prioridades principales con miras a la acción inmediata; el seguimiento de las tendencias, las reformas y las novedades; la promoción de la integración regional y la colaboración académica, mediante el apoyo a la creación y el desarrollo de ámbitos regionales de educación superior e investigación, y el fortalecimiento de las unidades regionales de la UNESCO en coordinación con las redes existentes;

g) fortalecer y ampliar el Equipo de Trabajo UNESCO-ADEA para la educación superior en África, en el que participan importantes asociados y donantes, con el fin de velar por el seguimiento eficaz de la Conferencia Mundial sobre la Educación Superior - 2009 y pasar así de las palabras a la acción.

Fonte: http://www.unesco.org/education/WCHE2009/comunicado_es.pdf 